\title{
Live whole-eye, ex vivo imaging and laser-induced micro injury of the corneal basal epithelium and visualization of resident macrophage responses
}

\author{
Sebastian M.D. Gulka ${ }^{1}$, Ana M. Litke ${ }^{1}$, Kerry. R. Delaney ${ }^{1}$, Robert L. Chow ${ }^{1}$ \\ ${ }^{1}$ University of Victoria, Department of Biology, Victoria, Canada
}

\begin{abstract}
Purpose. In this paper, we describe a novel live-imaging approach to visualize the short-term response of the mouse cornea to basal epithelial cell damage. Laser scanning confocal microscopy was used to induce precisely-defined, localized corneal basal epithelial cell damage and the live macrophage response to this damage was visualized and analyzed.

Methods. Lipophilic fluorescent dyes, SGC5 or FM 4-64, were injected into the anterior chamber of enucleated eyes and imaged live in whole-mount using confocal laser scanning microscopy. Laser-induced damage was performed by focusing onto a defined region of the corneal basal epithelium for a brief period using a high laser power setting and then returning to low laser power for imaging. Eyes from $C X 3 C R 1^{+/ G F P}$ mice were used to observe macrophage responses to laser damage in real-time.

Results. SGC5 or FM 4-64 dyes injected into the anterior chamber readily enter the cornea and are taken up by the stromal layer and labeled the outer membranes of corneal epithelial cells and remained stable when visualized using low laser power. Subjecting a defined region of the basal epithelium to high laser power for 1 minute or longer led to a rapid internalization of dye in the exposed basal epithelium cells and overall increase in cellular fluorescence. This change in fluorescence was also accompanied by cell swelling and contraction. Cellular internalization of the non-lipophilic, dye Alexa 647 hydrazide, indicated that membranes were compromised indicating that exposure to high power laser stimulation causes cellular damage to the basal epithelium. Visualization of corneal resident macrophages close to the site of laser-induced
\end{abstract}


damage showed that within minutes, projecting macrophage filopodia extended towards the damaged region at a rate of $0.75 \mu \mathrm{m} / \mathrm{min}$ for roughly 40 minutes.

Conclusion. We have developed a novel approach to image the live cornea and its response to damage. Laser-scanning confocal microscopy can be utilized to induce localized damage to mouse corneal basal epithelium and elicit a macrophage morphological response. This approach represents a useful tool for studying corneal wound healing and cellular responses to damage using live whole-mount imaging.

\section{Introduction}

The cornea is the transparent, complex arrangement of cells and connective tissue situated at the front of the eye. It serves a dual role in protecting the internal eye from external damage and in providing much of the refracting power of the eye. Because of the cornea's importance in vision, it is crucial that any damage it sustains is repaired in a manner that preserves its transparency. The corneal wound repair process is mediated by several cell types including keratocytes, epithelial cells, and macrophages (Wilson et al., 2001). In some disease states or corneal damage contexts, the wound healing process may cause hypervascularization and other abnormalities resulting in vision loss (Cursiefen et al., 2006).

Resident and infiltrating macrophages have been noted to exert several effects following corneal injury. Depending on the stage of the wound-healing process, macrophages can play both an inflammatory and an anti-inflammatory role (Italiani \& Boraschi, 2014; Krzyszczyk et al., 2018). Loss of either CCR2- or CCR2+ macrophages delays corneal wound healing as detected with fluorescein staining by about 6 hours (Liu et al., 2017a). Corneal macrophages play a key role in directly promoting corneal hem- and lymphangiogenesis following injury (Maruyama et al., 2005; Hos et al., 2016; Kiesewetter et al., 2019). Some of the effects of corneal macrophages in wound healing may be indirect. Interleukin-33 released by corneal macrophages is important in the induction of group 2 innate lymphoid cells which function in tissue repair (Liu, et al., 2017b). Autonomic nervous system input on macrophages affects their inflammatory phenotype, thus affecting the progression of wound healing (Xue et al., 2018). 
While our understanding of the role that macrophages play in corneal wound healing is growing, little has been reported on their acute physiologic response to small-scale damage. Previous methods aimed at damaging the cornea have used methods that cause widespread or extensive damage to the cornea including epithelial scraping (Liu et al., 2017a), suturing (Kiesewetter et al., 2019), and alkali burns (Kim et al., 2012). In addition, very few of the published experimental approaches have utilized live imaging to visualize corneal cellular responses to cellular damage.

Here, we report a novel live imaging, whole-mount approach to study corneal wound healing. Our approach utilizes lipophilic fluorescent dye labeling of corneal cell membranes can be visualized using confocal laser scanning microscopy (Litke et al., 2018). Focusing within the plane of the basal epithelium using high laser power leads to robust cellular damage to the exposed cells. Using this damage protocol in $C X 3 C R 1^{+/ G F P}$ mice enabled real time visualization of resident corneal macrophages and revealed rapid responses characterized by the formation of pseudopodia-like processes extending towards the site of damage.

\section{Methods}

\section{Mice}

All experiments were performed following approval by the University of Victoria Animal Care Committee in accordance with guidelines set by the Canadian Council for Animal Care. Mice on a 129S1 genetic background mice were used for laser-damage assay testing. $C X 3 C R 1^{+/ G F P}$ mice (Jung et al., 2000) were used for live and fixed imaging of Cx3crl-expressing immune cells. All animals were maintained on a 12-hour light/dark cycle and were 3-6 months of age.

\section{Anterior chamber injection}

Mice were euthanized using isoflurane followed by cervical dislocation. Eyes were enucleated and a pilot hole in the outer edge of the cornea was made using a 30-gauge beveled insulin syringe. A $50 \mu 1$ Hamilton glass syringe (model 705 RN SYR) with a 31-gauge, beveled needle was used to inject substances into the mouse anterior chamber (AC). When drawing up 
substances, care was taken to not draw up any air bubbles which would obscure imaging. Eyes were imaged within five minutes post injection.

\section{Confocal Microscopy}

For live whole mount imaging, enucleated mouse eyes were placed inside brain buffer (BB; 0.137M sodium chloride, $2.7 \mathrm{mM}$ potassium chloride, $2 \mathrm{mM}$ calcium chloride, $10 \mathrm{mM}$ HEPES

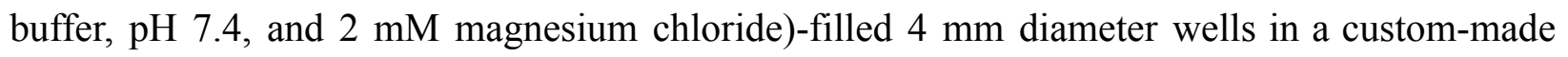
petri dish (made by pouring a small amount of Sylgard into a $35 \mathrm{~mm}$ petri dish and embedding the cut $\sim 5 \mathrm{~mm}$ end of a $0.6 \mathrm{~mL}$ centrifuge tube into the Sylgard before it dried). Eyes were imaged (without a coverslip) using a 60x water dipping lens (NA 1.0, WD $2.0 \mathrm{~mm}$, Nikon NIR APO 60x water objective, Nikon Corp.) For live imaging, z-stacks were set 1.0-1.5 $\mu \mathrm{m}$ apart. A $488 \mathrm{~nm}$ laser was used to image GFP and SGC5, $561 \mathrm{~nm}$ for FM 4-64, and $640 \mathrm{~nm}$ for Alexa 647 hydrazide. Live time-lapse images were imaged at a pixel density of $512 \times 512$ with a pixel dwell of 4.8. Imaging was performed using a Nikon C2 confocal microscope (Nikon Corp., Tokyo, Japan).

\section{Confocal laser-induced damage}

$2 \mu \mathrm{L}$ of $1 \mathrm{mM}$ Alexa 647 hydrazide (Cat. number A20502, Thermo Fisher, Waltham, MA) and 4 $\mathrm{uL}$ of $1 \mathrm{mM}$ of SGC5 (Cat. number 70057, Biotium Inc., Fremont, CA) was injected into the AC of an enucleated mouse eye. For $C X 3 C R 1^{+/ G F P}$ mice, only $4 \mu \mathrm{L}$ of $1 \mathrm{mM} \mathrm{FM} \mathrm{4-64} \mathrm{(Cat.} \mathrm{number}$ T13320, Thermo Fisher, Waltham, MA) was injected. Eyes were then imaged using confocal laser scanning microscopy. The $488 \mathrm{~nm}$ or $405 \mathrm{~nm}$ laser was used to damage eyes injected with SGC5, and the $561 \mathrm{~nm}$ laser of the confocal microscope for eyes injected with FM 4-64. The focus of the laser was set $6 \mu \mathrm{m}$ above the basal epithelial-stromal interface. The field of view was zoomed in $6 \mathrm{x}$ to scan a region of $1600 \mu \mathrm{m}^{2}$ and the laser power setting was turned to maximum (100.00). For epithelial damage assays, lasers were run for 60 seconds at various laser power settings. For macrophage response experiments, the laser was scanned continuously for 10 minutes at a resolution of 512 x 512 pixels and pixel dwell was set to $4.8 \mu \mathrm{sec}$. The area surrounding the damage was observed for 2-2.5 hours after the damage stimulus using a low laser power setting (2.0). 


\section{Image analysis}

\section{Image Adjustment}

Images were analyzed using the Fiji suite of ImageJ (Schindelin et al., 2012). This included 3D drift correction for time-lapse images, pixel intensity adjustments, background subtraction, and distance measurements.

\section{Quantification of cell dynamics}

ImageJ was used to quantify cell dynamics of both steady-state and injury-responding cells. Time-lapse images were first corrected using plugins $>$ registration $>$ correct $3 \mathrm{D}$ drift. A maximum projection of the cell(s) of interest was generated. When measuring length change of cell processes, the first timepoint captured was used as the reference position. A perpendicular line was drawn through the base of the process in the initial reference position and a spot was marked in the middle of that line as the origin to measure from. Lengths were measured from the reference point of the specified cell process to the furthest edge of the cell process in the next timepoint.

\section{Results}

\section{Confocal laser induces basal epithelial cell damage}

In order to visualize corneal cell types in live wholemount preparations, we injected the lipophilic dye SGC5 into the anterior chamber of enucleated eyes. (Figure 1A). We found that SGC5 diffused readily through the stroma and labeled the outer membrane of basal epithelial cells (Figure 1A) and corneal wing cells (data not shown). When imaged at low laser power, membrane labeling of the epithelial cells remained stable and unchanged for $>30$ minutes.

To induce damage, corneas were exposed to either the $405 \mathrm{~nm}$ or $488 \mathrm{~nm}$ laser line set at high power settings and focussed on a single plane set $6 \mu \mathrm{m}$ above the basal epithelial-stromal interface (Figure 1C). This exposure led to an overall increase in basal cell fluorescence, apparent internalization of fluorescence as early as 3 minutes after the onset of the high laser 
power (Figure 2). After 10 minutes of exposure, cell swelling followed by contraction was evident in many of the basal epithelial cells early (Figure 2, see cyan colored cell). Similar responses were also observed with as little as 1 minute of exposure to high power laser scanning (Figure 3D). Basal epithelial cells in corneas injected with either FM 4-64 or SGC5 responded identically to laser damage (Figure 3A).

As the changes in FM 4-64 or SGC5 fluorescence and cell morphology observed following high power laser exposure suggested that the basal epithelium was being damaged, we next wanted to determine whether there were also changes in cell permeability, which is a sign of cell damage (Ünal-Çevik et al., 2004; reviewed in Elmore, 2007). To examine this, eyes were co-injected with the hydrophilic dye, Alexa 647 hydrazide. Similar to SGC5, Alexa 647 hydrazide efficiently entered the stroma, but did not penetrate into the epithelium (data not shown). Robust filling of individual basal epithelial cells with Alexa 647 hydrazide was observed in laser-damaged cells after about 20 minutes after the onset of high power laser exposure, suggesting a potential rupture of the cell membrane (Figure 3B,C). A laser-power titration revealed that the intensity of SGC5 fluorescence and presence of Alexa 647 hydrazide in basal epithelial cells increased as the laser power setting increased, with Alexa 647 hydrazide first penetrating into cells at a setting of 23.0 out of 100.0 (Figure 3B). These data reveal a non-lytic membrane damaging of basal epithelial cells by confocal laser, with no obvious damage to the overlying superficial epithelium.

\section{CX3CR1+ cells response to laser-induced damage}

To observe whether the laser-induced basal epithelial damage was sufficient to induce a response in the stromal-localized, resident macrophage population, the enucleated eyes of $C X 3 C R 1^{+/ G F P}$ mice were subjected to the same laser-damage technique mentioned above. Time-lapse videos of undamaged ex vivo corneas revealed $C X 3 C R 1^{+/ G F P}$ cells extending and retracting filopodia in a stochastic manner (data not shown). Following laser-induced damage to basal epithelium, nearby macrophages extended pseudopodia to the damaged region (Figure 4). While macrophage pseudopodia exhibited stochastic movements in the naive cornea, following laser damage to nearby basal epithelium, the pseudopodia bud closest to the damage grew markedly in length, while others remained constant, shortened slightly or retract completely to leave the cell in a highly polarized state (Figure 5A,B). There was no evidence that macrophages were migrating 
toward the site of damage (data not shown). Pseudopodia-like projections from responding macrophages approached and entered the damaged region, reaching full extension roughly 40 minutes after the laser-damage ended (Figure 5C,D). Once pseudopodia reached full extension, morphological changes were not observed in the responding cells for the remaining observation time. Comparing multiple macrophage responses led to a similar trend of an initial filopodia growing phase from 0 minutes to 40 minutes, with an average extension rate of $0.75 \mu \mathrm{m} / \mathrm{minute}$ for the first 30 minutes (Figure 5D).

\section{Discussion}

In this study, a novel corneal basal epithelial damage method laser was used, and the live response to damage was captured. The laser-damaged basal epithelium elicited a macrophage response. While lasers have been used in other tissues to cause damage leading to a macrophage/microglial response (Davalos et al., 2005; Taylor et al., 2018), there have been no studies that investigate the live corneal macrophage response to damage until now. As the damage in our approach was focussed on the basal epithelium, the superficial epithelium was not damaged, and cells were not lysed. Alexa 647 hydrazide penetration into damaged epithelial cells indicates that cell membranes were compromised. Our damage-inducing laser method appears similar to that used by Septiadi et al. (2018), who used a confocal scanning laser to cause pulmonary epithelial cell injury. In their case cell death was determined to be the result of apoptosis, with cells maintaining adhesive properties after irradiation.

Both Davalos et al. (2005) and Taylor et al. (2018) used laser ablation to induce an injury in the mouse brain and observed a microglial response similar to our macrophage response, with responding cells sending processes to the damaged region within minutes of injury. In our study, corneal $C x 3 \operatorname{crl}: G F P+$ cells responded to damage on a slightly slower timescale than microglia in the brain, not reaching full extension until 30-60 minutes post-injury in laser-damaged corneas. As excess inflammation may cause corneal defects due to abnormal healing (Matsuda \& Smelser, 1973), this may be an intentional way to mitigate those outcomes. The slower response may also be due to the relatively lower density of macrophages in the cornea or the complexity of the stroma preventing efficient diffusion of damage-associated molecular patterns. The 
corneal macrophage response observed here also appears similar to that seen by Uderhardt et al. (2019), with responding cells projecting one or more pseudopodia-like processes towards a laser-damaged damaged region.

While wound-infiltrating macrophages have been noted to play only a minor role in promoting inflammation (Oshima et al., 2006), resident macrophages have a significant role in wound evolution. Uderhardt et al. (2019) found that resident tissue macrophages in the peritoneum act to cloak small injuries and prevent the influx of neutrophils, which exacerbate tissue damage and inflammation. Given the similarity of our observed macrophage response to theirs, this may be what we observed. Further studies including neutrophil observation are required to confirm this.

Altogether this study shows that fine damage to the corneal epithelium is enough to elicit a stromal macrophage response. Laser-induced injury leads to a localized damage of corneal basal epithelial cells. While the exact nature of this response has yet to be determined, the initial observation of filopodia extension may indicate a cloaking, probing, or phagocytic action. Further studies in the cornea could focus on comparing different types of damage including and beyond what was tested here to determine the threshold for macrophage activation or to see how other immune cells (infiltrating or resident) respond and observing longer timepoints in vivo to determine the result of the macrophage response.

\section{Acknowledgements}

This work was funded in part by funding from the National Sciences and Engineering Research Council of Canada and the Canadian Institute for Health Research to RLC. 


\section{References}

Brissette-Storkus, C.S., Reynolds, S.M., Lepisto, A.J., \& Hendricks, R.L. 2002. Identification of a novel macrophage population in the normal mouse corneal stroma. Investigative Ophthalmology and Visual Science. 43(7): 2264-2271.

Chan, K.Y., Cho, P., \& Boost, M. 2014. Corneal epithelial cell viability of an ex vivo porcine eye model. Clinical and Experimental Optometry. 97: 337-340. doi: $10.1111 /$ cxo. 12128

Chinnery, H.R., Ruitenberg, M.J., Plant, G.W., Pearlman, E., Jung, S., \& McMenamin, P.G. 2007. The chemokine receptor CX3CR1 mediates homing of MHC class II-positive cells to the normal mouse corneal epithelium. Investigative Ophthalmology and Visual Science. 48(4): 1568-1574.

Chinnery, H.R., Humphries, T., Clare, A., Dixon, A.E., Howes, K., Moran, C.B., Scott, D., Zakrzewski, M., Pearlman, E., \& McMenamin, P.G. 2008a. Turnover of bone marrow-derived cells in the irradiated mouse cornea. Immunology. 125: 541-548.

Chinnery, H.R., Pearlman, E., \& McMenamin, P.G. 2008b. Cutting edge: membrane nanotubes in vivo: a feature of MHC class II+ cells in the mouse cornea. The Journal of Immunology. 180(9): 5779-5783. doi:10.4049/jimmunol.180.9.5779

Choy, E.P.Y., To, T.S.S., Cho, P., Benzie, I.F.F.D., \& Choy, C.K.M. 2004. Viability of porcine corneal epithelium ex vivo and effect of exposure to air: a pilot study for a dry eye model. Cornea. 23(7): 715-719. doi:10.1097/01.ico.0000127475.29551.56

Cursiefen, C., Maruyama, K., Jackson, D.G., Streilein, J.W., \& Kruse, F.E. 2006. Time course of angiogenesis and lymphangiogenesis after brief corneal inflammation. Cornea. 25(4): 443-447. doi: 10.1097/01.ico.0000183485.85636.ff.

Davalos, D., Grutzendler, J., Yang, G., Kim, J.V., Zuo, Y., Jung, S., Littman, D.R., Dustin, M.L., \& Gan, W. 2005. ATP mediates rapid microglial response to local brain injury in vivo. Nature Neuroscience. 8(6): 752-758. doi: 10.1038/nn1472

Domingo, E., Moshirfar, M., \& Zabbo, C.P. 2020. Corneal Abrasion. StatPearls. Treasure Island (FL), StatPearls Publishing.

Elmore, S. 2007 Apoptosis: A review of programmed cell death. Toxicologic Pathology. 35(4): 495-516. doi: 10.1080/01926230701320337

Hos, D., Bucher, F., Regenfuss, B., Dreisow, M., Bock, F., Heindl, L.M., Eming, S.A., \& Cursiefen, C. 2015. The American Journal of Pathology. 186(1): 159-171. doi: 10.1016/j.ajpath.2015.09.012. 
Italiani, P., \& Boraschi, D. 2014. From monocytes to M1/M2 macrophages: phenotypical vs. functional differentiation. Frontiers in Immunology. 5:514. doi: 10.3389/fimmu.2014.00514

Jung, S., Aliberti, J., Graemmel, P., Sunshine, M.J., Kreutzberg, G.W., Sher, A., \& Littman, D.R. 2000. Analysis of fractalkine receptor CX(3)CR1 function by targeted deletion and green fluorescent protein reporter gene insertion. Molecular Cell Biology. 20: 4106-4114. doi: 10.1128/MCB.20.11.4106-4114.2000

Kiesewetter, A., Cursiefen, C., Eming, S.A., \& Hos, D. 2019. Phase-specific functions of macrophages determine injury-mediated corneal hem- and lymphangiogenesis. Scientific Reports. 9, 308.

Kim, E.C., Kim, T.K., Park, S.H., \& Kim, M.S. 2012. The wound-healing effects of vitamin A eye drops after a corneal alkali burn in rats. Acta Ophthalmologica. 90(7): e540-546. doi: 10.1111/j.1755-3768.2012.02496.x

Knickelbein, J.E., Watkins, S.C., Mcmenamin, P.G., \& Hendricks, R.L. 2009. Stratification of antigen-presenting cells within the normal cornea. Ophthalmology and Eye Diseases. 1: 45-54.

Krzyszczyk, P., Schloss, R., Palmer, A., \& Berthiaume, F. 2018. The role of macrophages in acute and chronic wound healing and interventions to promote pro-wound healing phenotypes. Frontiers in Physiology. 9(419). doi: 10.3389/fphys.2018.00419

Lee, M., Lee, Y., Song, J., Lee, J., Chang, S. 2018. Tissue-specific role of CX3CR1 expressing immune cells and their relationships with human disease. Immune Network. 18(1):e5. doi: 10.4110/in.2018.18.e5

Liu, J., Xue Y., Dong, D., Xiao, C., Lin, C., Wang, H., Song, F., Fu, T., Wang, Z., Chen, J., Pan, H., Li, Y., Cai, D., \& Li, Z. 2017a. CCR2 ${ }^{-}$and CCR2 ${ }^{+}$corneal macrophages exhibit distinct characteristics and balance inflammatory responses after epithelial abrasion. Mucosal Immunology. 10(5):1145-1159. doi: 10.1038/mi.2016.139

Liu, J., Chengju, X., Wang, W., Xue, Y., Dong, D., Lin, C., Song, F., Fu, T., Wang, Z., Chen, J., Pan, H., Li, Y., Cai, D., \& Li, Z. 2017b. Local group 2 innate lymphoid cells promote corneal regeneration after epithelial abrasion. The American Journal of Pathology. 187(6): 1313-1326. doi: 10.1016/j.ajpath.2017.02.010

Litke, A.M., Samuelson, S., Delaney, K.R., Sauvé, Y., Chow, R.L., 2018. Investigating the pathogenicity of VSX1 missense mutations and their association with corneal disease. Investigative Ophthalmology and Visual Science. 59(15): 5824-5835. 
Matsuda, H., \& Smelser, G.K. 1973. Epithelium and stroma in alkali-burned corneas. Archives of Ophthalmology. 89: 396-401. doi: 10.1001/archopht.1973.01000040398009

Maruyama, K., Li, M., Cursiefen, C., Jackson, D.G., Keino, H., Tomito, M., Van Rooijen, N., Takenaka, H., D’Amore, P.A., Stein-Streilein, J., Losordo, D.W., \& Streilein, J.W. 2005. Inflammation-induced lymphangiogenesis in the cornea arises from CD11b-positive macrophages. The Journal of Clinical Investigation. 115(9): 2363-2372. doi: https://doi.org/10.1172/JCI23874.

Maurice, D.M. 1957. The structure and transparency of the cornea. The Journal of Physiology. 136: 263-286.

Oshima, T., Sonada, K-H., Tsutsumi-Miyahara, C., Qiao, H., Hisatomi, T., Nakao, S., Hamano, S., Egashira, K., Charo, I.F., Ishibashi, T. 2006. Analysis of corneal inflammation by cauterisation in CCR2 and MCP-1 knockout mice. British Journal of Ophthalmology. 90: 218-222. doi: 10.1136/bjo.2005.077875

Septiadi, D., Abdussalam, W., Rodriguez-Lorenzo, L., Spuch-Calvar, M., Bourquin, J., Petri-Fink, A., \& Rothen-Rutishauser, B. 2018. Revealing the role of epithelial mechanics and macrophage clearance during pulmonary epithelial injury in the presence of carbon nanotubes. Advanced Materials. 30(52). doi: 10.1002/adma.201806181

Schindelin, J., Argando-Carreras, I., Frise, E., Kaynig, V., Longair, M., Pietzsch, T., Preibisch, S., Rueden, C., Saalfeld, S., Schmid, B., Tinevez, J., White, D.J., Hartenstein, V., Eliceiri K., Tomancak, P., \& Cardona, A. 2012. FijiL an open-souce platform for biological image analysis. Nature Methods. 9: 676-682. doi: 10.1038/nmeth.2019

Taylor, S., Mehina, E., White, E., Reeson, P., Yongblah, K., Doyle, K.P., \& Brown, C.E. 2018. Suppressing interferon- $\gamma$ stimulates microglial responses and repair of microbleeds in the diabetic brain. The Journal of Neuroscience. 38(40): 8707-8722. doi: 10.1523/JNEURSCI.0734-18.2018

Uderhardt, S., Martins, A.J., Tsang, J.S., Lämmerman, T., \& Germain, R.N. 2019. Resident macrophages cloak tissue microlesions to prevent neutrophil-driven inflammatory damage. Cell. 177: 541-555. doi:

https://doi.org/10.1016/j.cell.2019.02.028

Ünal-Çevik, Kilinç, M., Can, A., Gürsoy-Özdemir, \& Dalkara, T. 2004. Apoptotic and necrotic death mechanisms are concomitantly activated in the same cell after 
cerebral ischemia. Stroke. 35(9): 2189-2194. doi:

10.1161/01.STR.0000136149.81831.c5

Wilson, S.E., Mohan, R.R., Mohan, R.R.Ambósio Jr., R., Hong, J., \& Lee, J. 2001. The corneal wound-healing response: cytokine-mediated interaction of the epithelium, stroma, and inflammatory cells. Progress in Retinal and Eye Research. 20(5): 625-637. doi: 10.1016/s1350-9462(01)00008-8.

Xue, Y., He, J., Xiao, C., Guo, Y., Fu, T., Liu, J., Lin, C., Wu, M., Yang, Y., Dong, D., Pan, H., Xia, C., Ren, L., \& Li, Z. 2018. The mouse autonomic nervous system modulates inflammation and epithelial renewal after corneal abrasion through the activation of distinct local macrophages. Mucosal Immunology. 11:1496-1511. doi: $10.1038 /$ s41385-018-0031-6 


\section{Figure Legends}

Figure 1. Overview of anterior chamber injection and laser damage protocol. (A) Schematic of cornea and anterior chamber injection. Alexa 647 hydrazide and either SGC5 or FM 4-64 (orange in diagram) were injected into the anterior chamber and diffused into the corneal. SGC5 and FM 4-64 labeled cell membranes while Alexa647 hydrazide was confined to the stroma. (B) SGC5 labeling in the corneal stroma and basal epithelium. (C) Cornea laser-damage protocol. Eyes from euthanized mice were enucleated, injected, and a small region of cornea was imaged at high power for 10 minutes. epi = corneal epithelium, b. epi $=$ basal epithelium, $\mathrm{S}=$ superficial epithelium, $\mathrm{W}=$ wing cells, $\mathrm{B}=$ basal epithelium. Scale $\mathrm{bar}=30 \mu \mathrm{m}$.

Figure 2. Laser-damaged basal epithelium. Corneas were imaged for 30 minutes with a low-power $488 \mathrm{~nm}$ laser which caused no change in membrane dye labeling. The basal epithelium was then co-stimulated with a high-power $405 \mathrm{~nm}$ laser for 10 minutes. Within 3 minutes, fluorescence was observed within cells, and membrane fluorescence increased. Laser-damaged epithelial cells began swelling followed by contraction within 40 minutes of the onset of the $405 \mathrm{~nm}$ laser. A cell undergoing swelling and contraction is highlighted In blue. Times are minutes since onset of $405 \mathrm{~nm}$ laser. Scale bar $=7.5 \mu \mathrm{m}$

Figure 3. Confocal laser damage effect on basal epithelium of eyes injected SGC5 and Alexa 647 hydrazide dyes. (A) Basal epithelium of corneas injected with FM 4-64 or SGC5 lipophilic membrane dyes behaved similarly after laser damage (B) Alexa 647 hydrazide enters laser-damaged cells at 23.0 laser power setting, while SGC5 intensity increased linearly with laser power. (C) Imaging of corneal basal epithelium from B. The laser power setting for each damaged region is indicated on the corresponding square in the leftmost image. (D) Time-course of corneal basal epithelium damaged with 60 laser-power setting for 60 seconds. Alexa 647 hydrazide penetrates damaged cells 20 minutes-post laser. Scale bar $=30 \mu \mathrm{m}(\mathrm{A}), 15 \mu \mathrm{m}(\mathrm{C})$.

Figure 4. Macrophage response to laser-damaged basal epithelium. (A) When nearby basal epithelium is damaged, macrophages project filopodia to the laser-damaged region (blue square). FM 4-64 (from anterior chamber injection) labels cell membranes, while GFP is from Cx3crl:GFP+ cells. Times are minutes after offset of high powered laser exposure. Scale bar $=$ $30 \mu \mathrm{m}(\mathrm{A})$ 
Figure 5. Response of multiple macrophages to laser-damaged basal epithelium. (A) Damage-responding macrophage before and after damage. Labeled processes 1-4 corresponding to the graph in B. (B) Data from the macrophage shown in A reveals that the filopodia closest to the damaged area (process 1) reached full extension after 30 minutes, while other filopodia decreased. Red line indicates time of injury. (C) Live imaging of multiple macrophage responses to laser damage (blue square). Macrophages extended filopodia (yellow arrowhead) to the damaged region. (D) Graph of six responding macrophages from A and C revealed an average extension rate of $0.75 \mu \mathrm{m} / \mathrm{min}$. Times indicated are minutes after offset of high powered laser exposure. Scale bar $=20 \mu \mathrm{m}(\mathrm{A})$ and $30 \mu \mathrm{m}(\mathrm{C})$. 
A
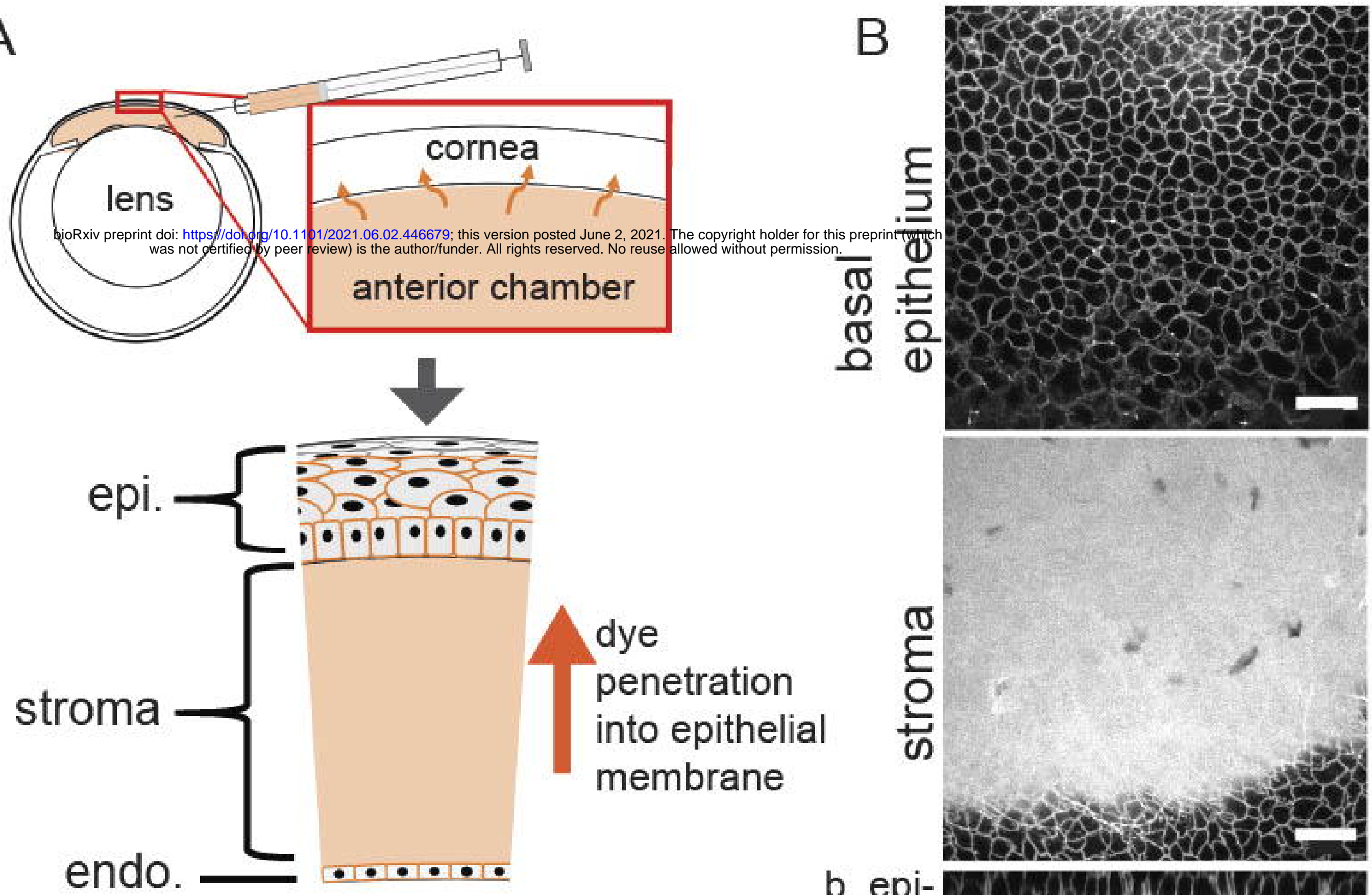

dye penetration into epithelial membrane

endo. $\square$

b. epi-

stroma-

C

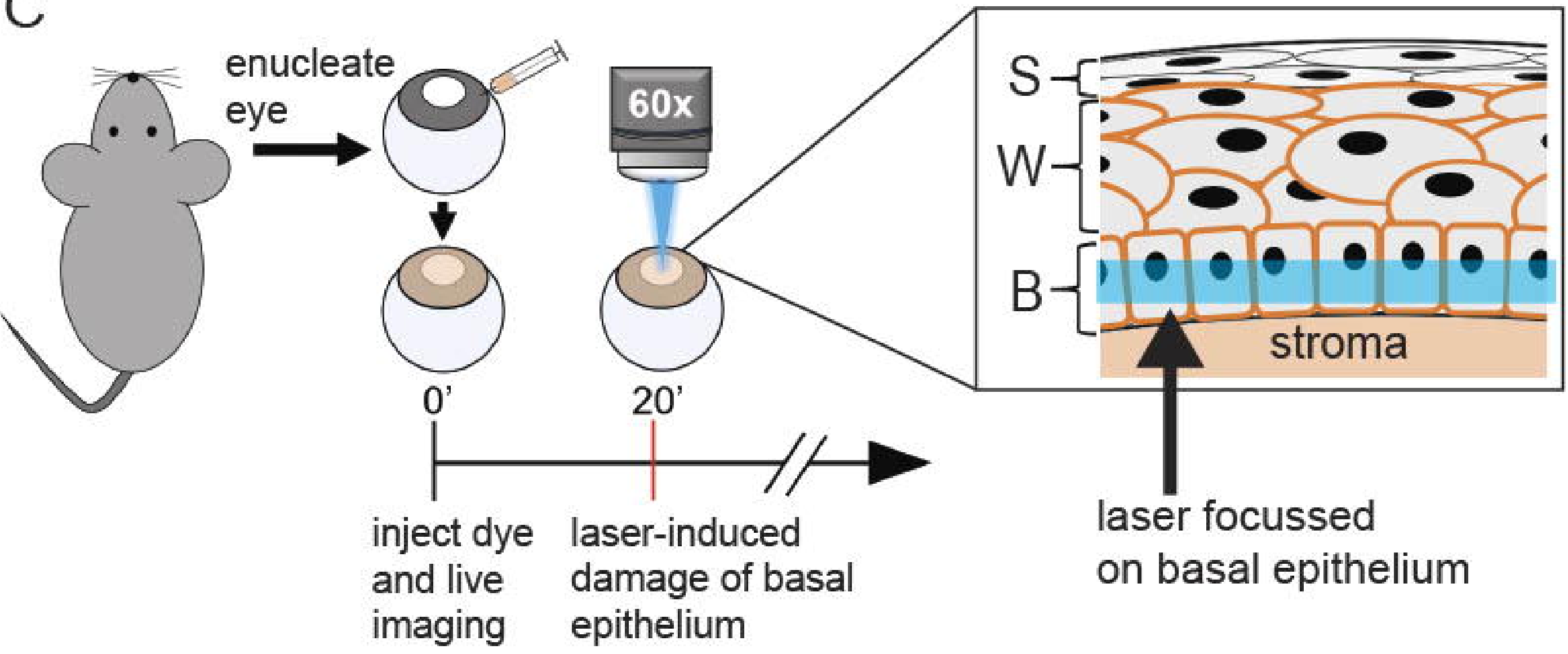




\section{5 hi laser power ( 0 ' to $10^{\prime}$ )}
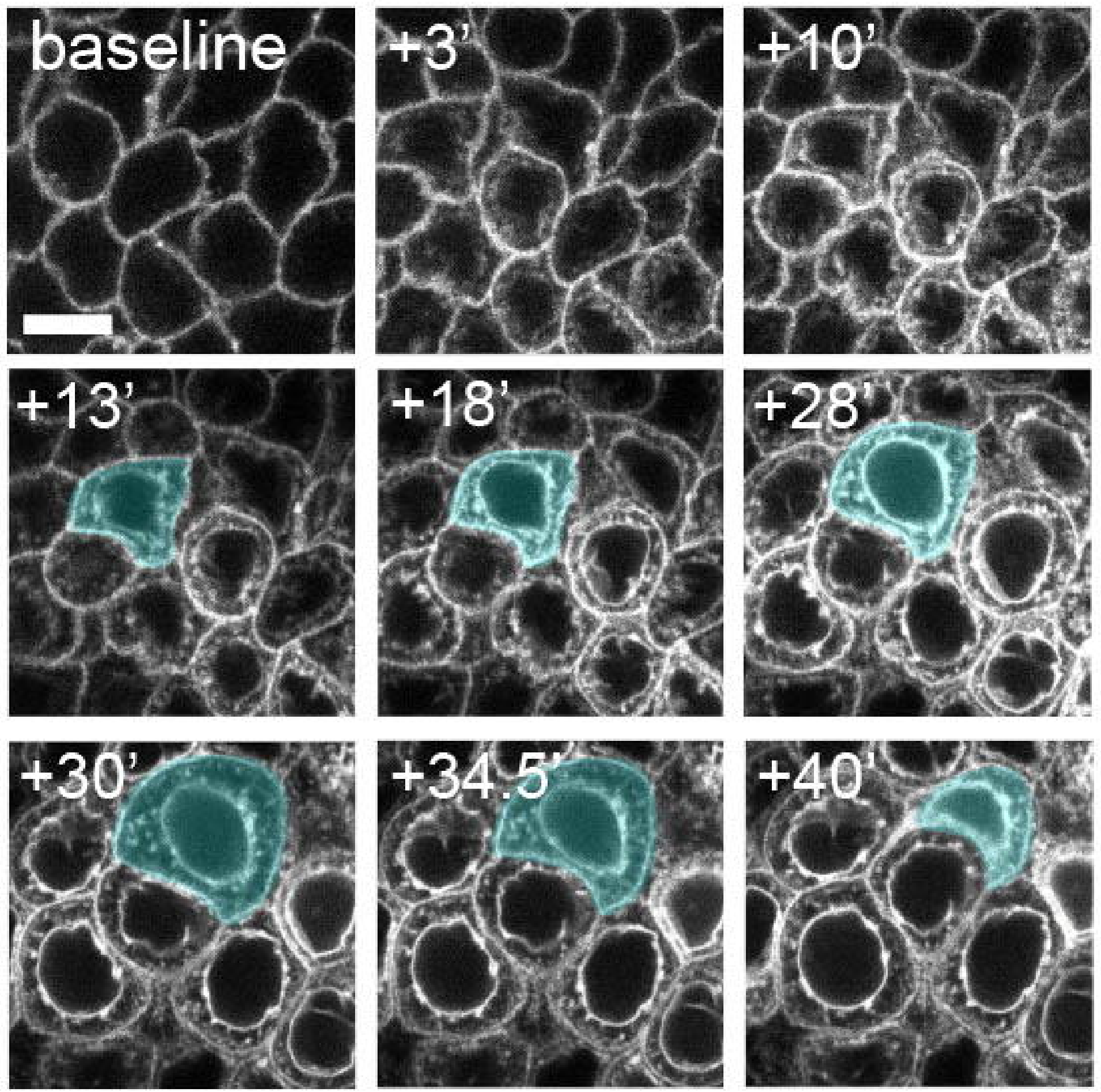
A

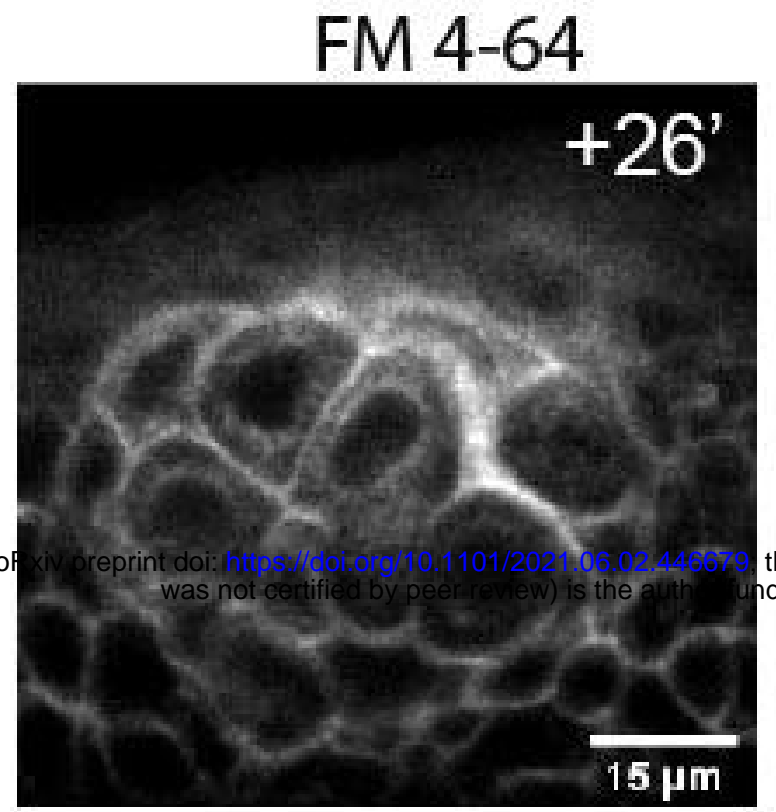

C

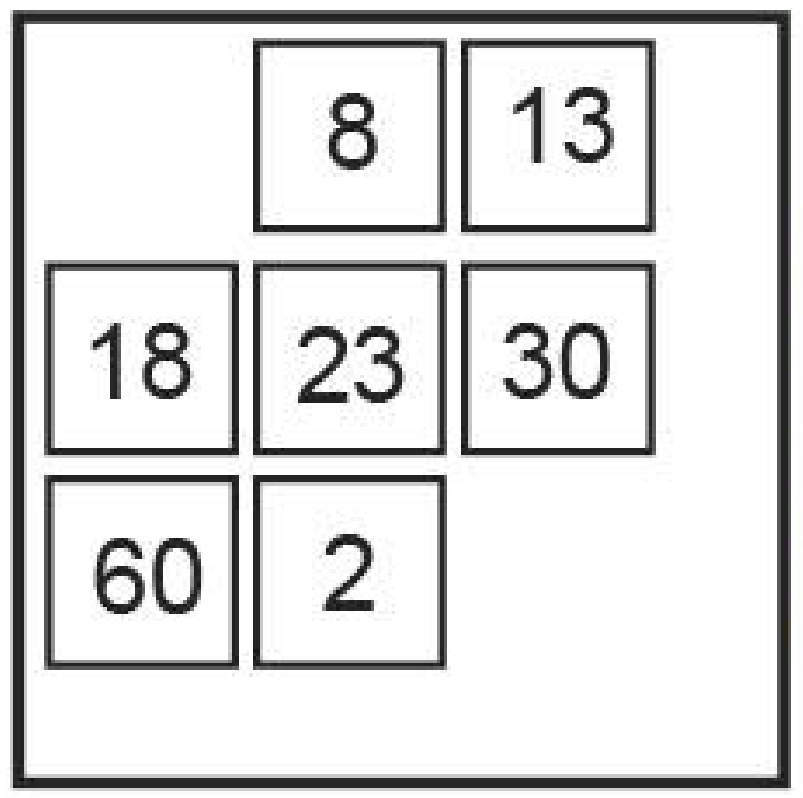

D

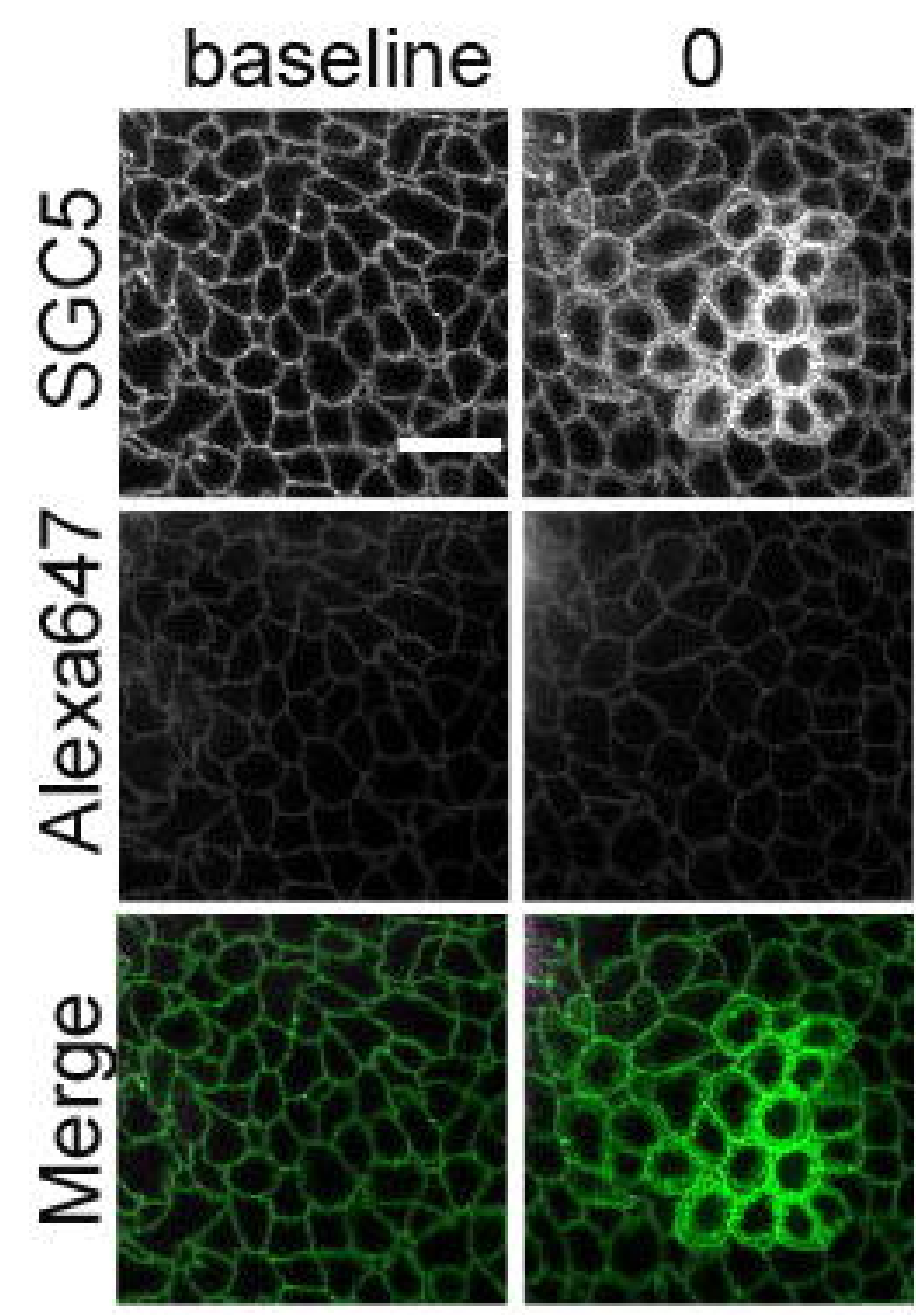

SGC5

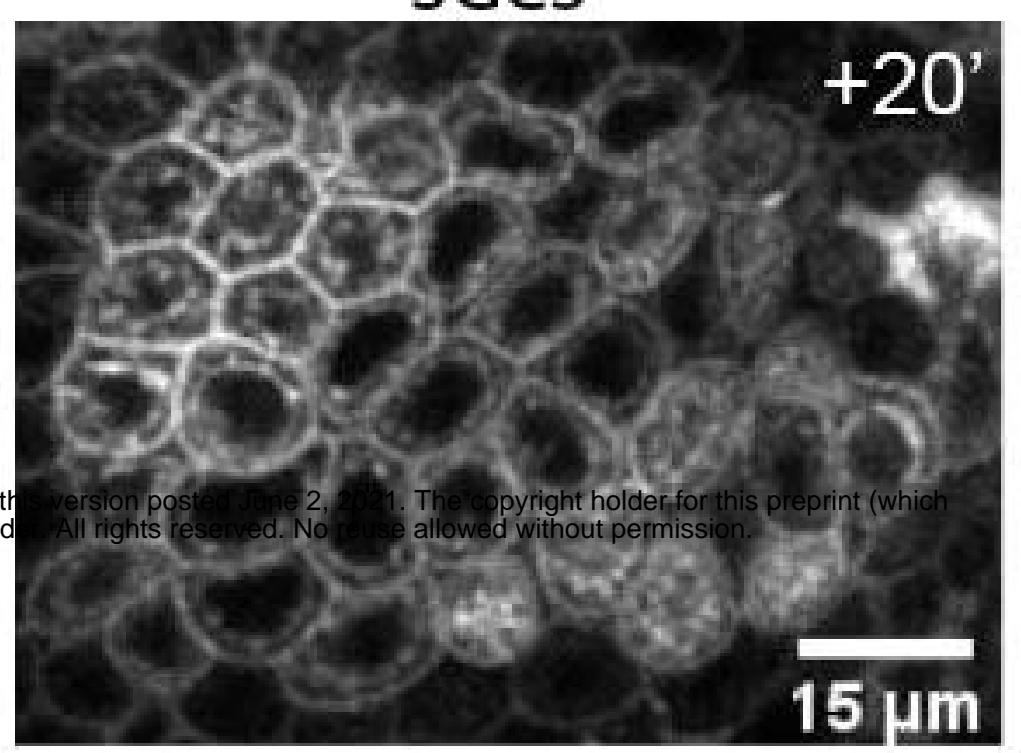

B
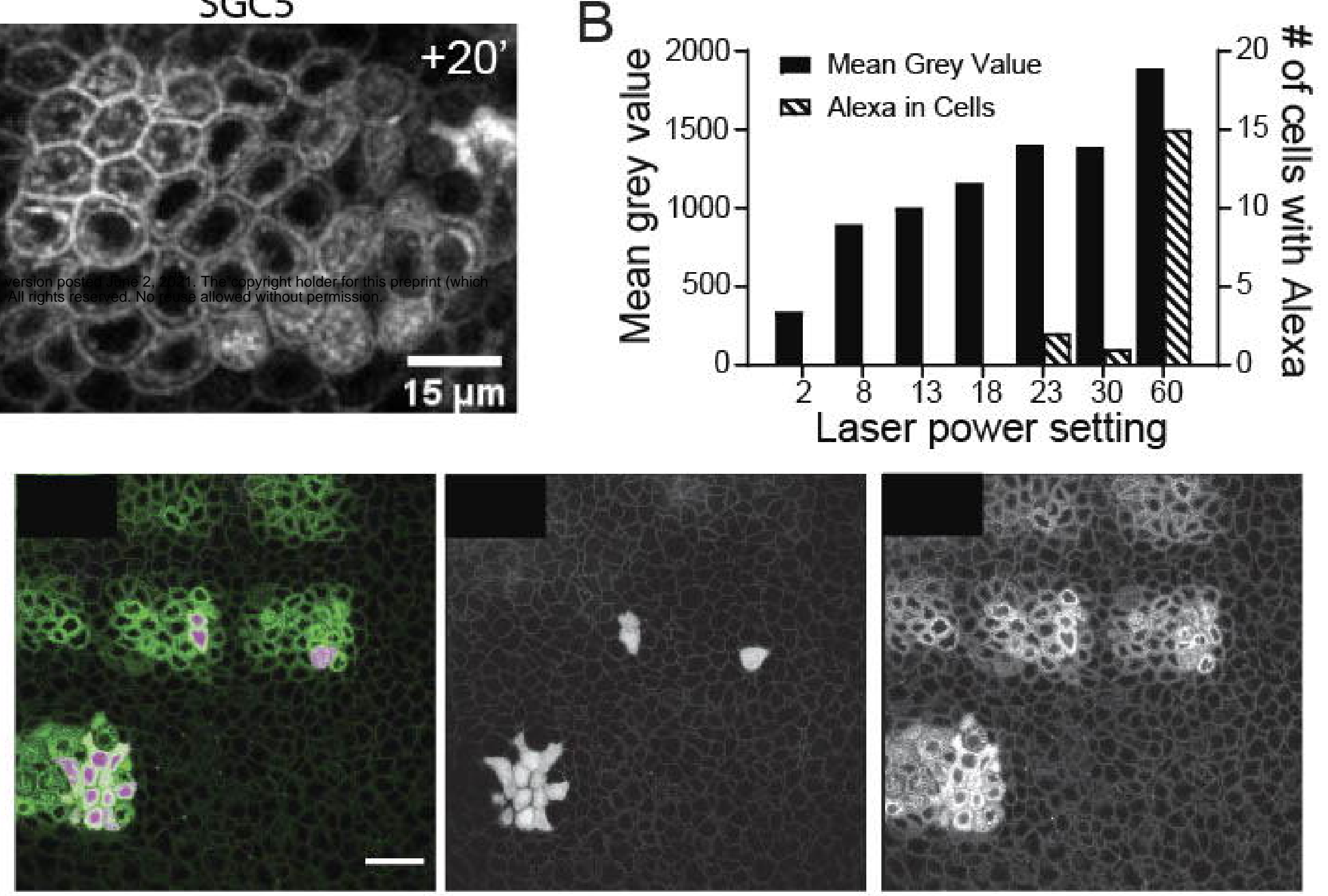

Time after damage (min)
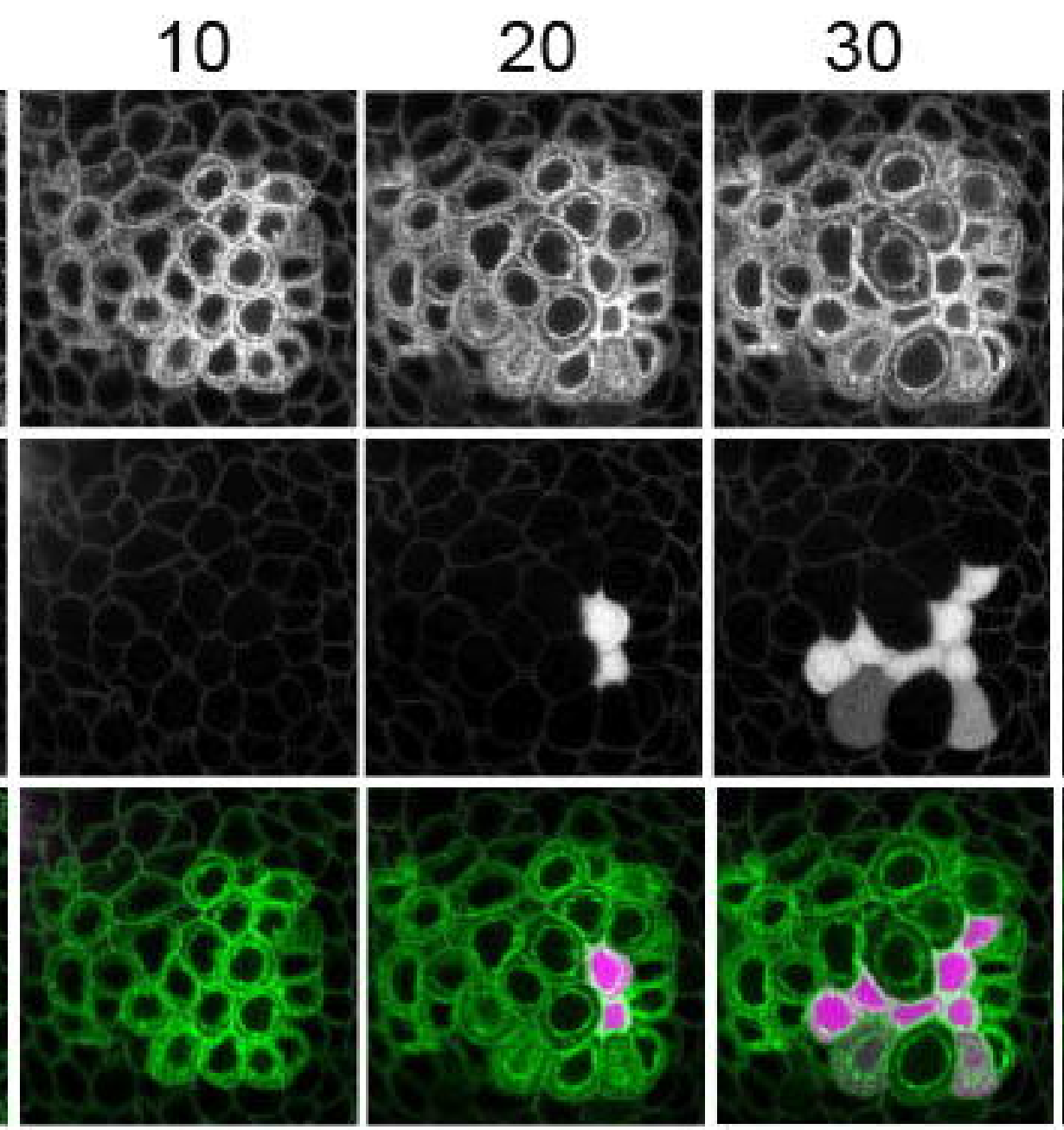

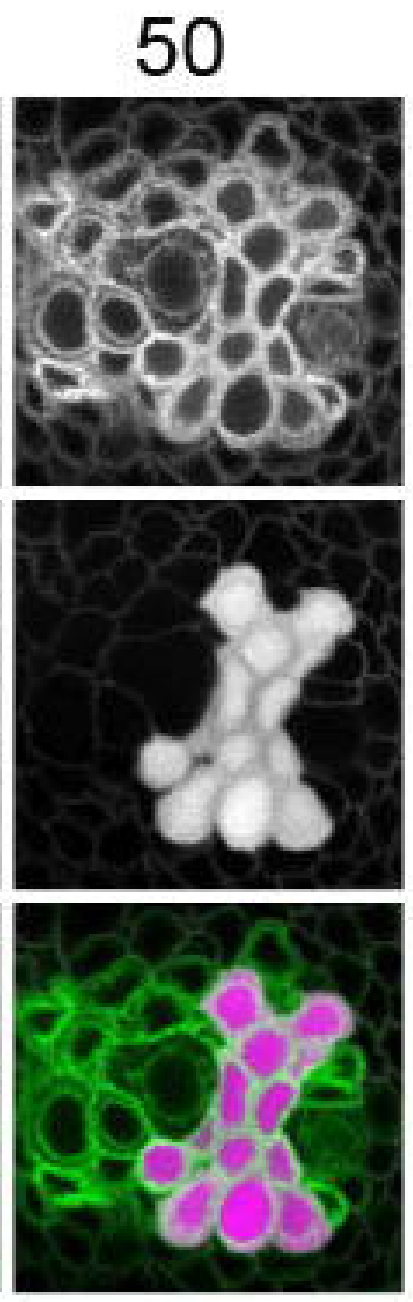




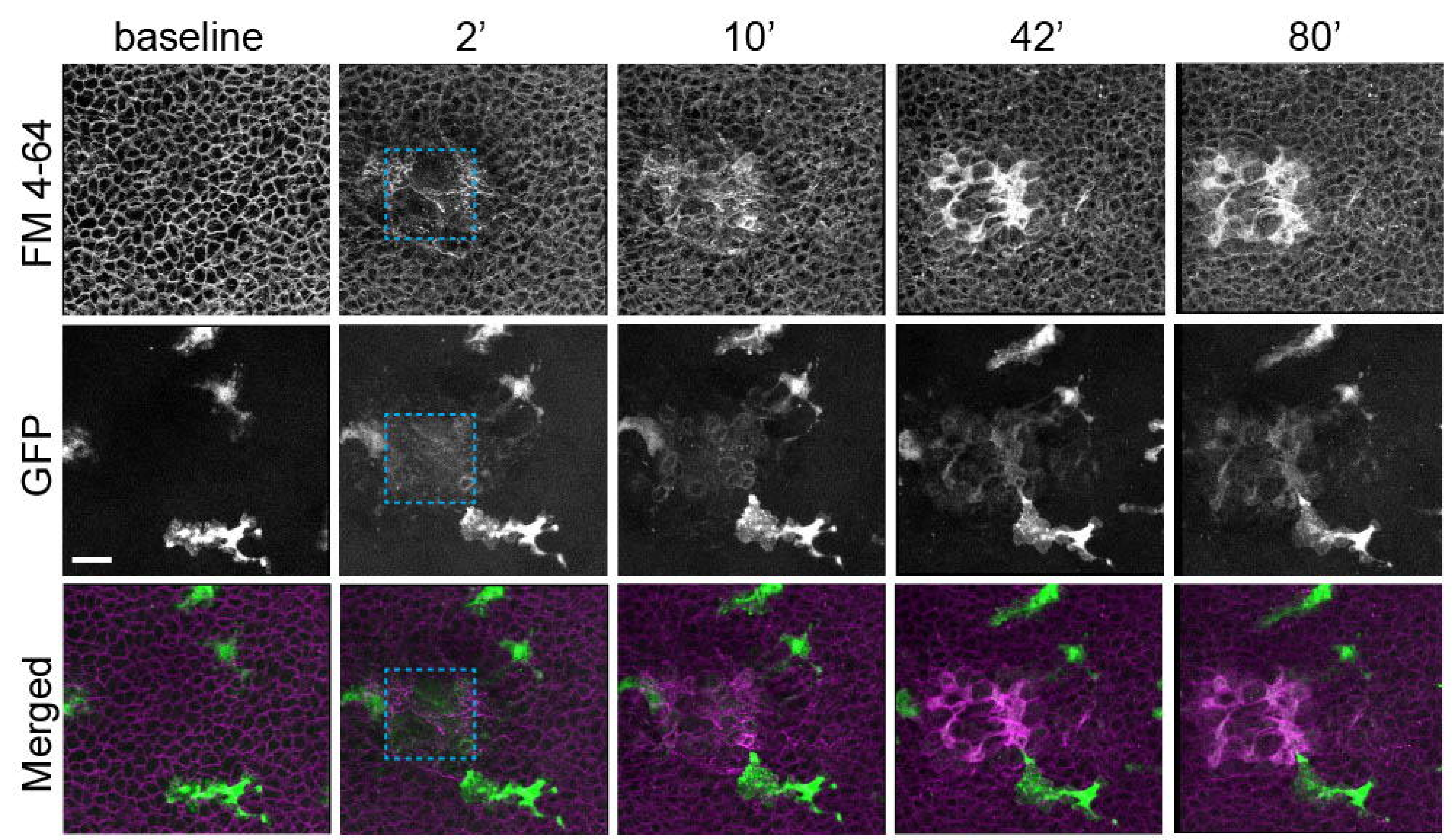




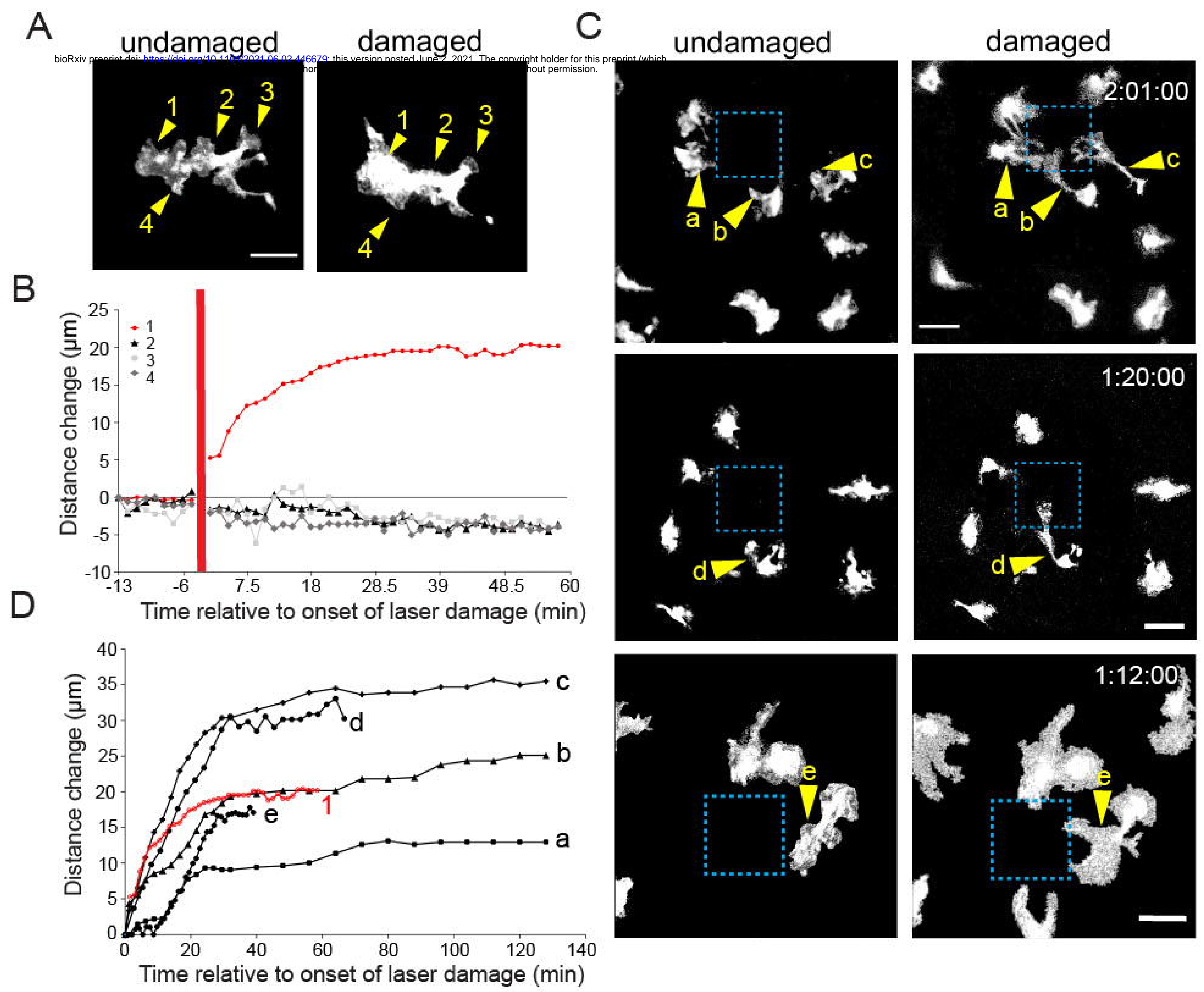

